\title{
Determining internal forces in modular building elements under action wind load
}

\author{
Viacheslav Shirokov ${ }^{1, *}$, Alexey Soloviev ${ }^{1}$, and Tatiana Gordeeva ${ }^{1}$ \\ ${ }^{1}$ Samara State Technical University, Academy of Architecture and Civil Engineering, \\ Molodogvardeyskaya St., 194, Samara, 443001, Russia
}

\begin{abstract}
The research paper focuses on internal forces determination in the elements of modular buildings under wind load. It provides a methodology for determining dynamic characteristics of a building and for calculating wind loads. This method is based on the following assumptions: coupling of the modules elements is rigid; coupling of blockmodules with foundations is hinged-fixed; connection of blocks to each other is hinged in angular points; the floor disk in its plane is not deformed. On the basis of these assumptions the authors derived approximate and refined equations for determining forces in modules elements under static and pulsation components of wind load. The equation of bending moments determination in the pillar bearing cross-section is obtained by approximation of the graph of moments variation, calculated for the spectrum of the ratio of the pillar stiffness and the floor beam in the range from 1/64 to 64 . The paper further introduces the calculation results of forces based on the proposed methodology and on the finite element method. The calculations were done while taking different values of wind load and different number of storeys in a building (from 1 to 4 floors). The obtained results are similar, the error does not exceed 5\%.
\end{abstract}

\section{Introduction}

At present, modular buildings are actively used in construction. Modular technology is relatively new in our country. That is why many researchers began to study such buildings [1-7]. Most of the research works consider technological, economic and architectural components, and all the authors conclude that the modular construction technology is promising. At the same time, there are very few works devoted to the static and dynamic work of modular buildings. In articles $[8,9]$ it is shown that static and dynamic operation study of modular buildings is a currently important direction. The relevance of such studies is determined by the following reasons:

- modular buildings application on territories with harsh climate and complex seismic conditions;

- tridimentional structural behavior;

- the lack of regulatory framework for modular building design.

In article [8] it was shown that the wind load can have a significant effect on the stressstrain behavior (SSB) of the modular building. The most common method for determining 
wind load and effort from it is the numerical finite element method (FEM), since it is the most universal one. However, the FEM has the following drawbacks:

- it is necessary to have complete information on the structure stiffness and its geometry;

- in most cases it is necessary to create a tridimentional design model of construction.

These drawbacks lead to the fact that at the stage of pre-design decision making about the modular building construction, it takes considerable time to create a design model, and for unknown elements cross-sections to create several models. Also with the help of FEM it is difficult to establish general patterns of static and dynamic operation of the construction, which is necessary for their SSB study. Therefore, it is advisable to have an analytical technique for determining the wind load on the building and the effort from it.

\section{Materials and methods}

\subsection{Wind load determination on a modular building}

In general, the design model of one block-module represents a three dimentional frame, all the elements of which are rigidly connected to each other, the hinged immovable supports is located at the corner points at the bottom (Figure 1).

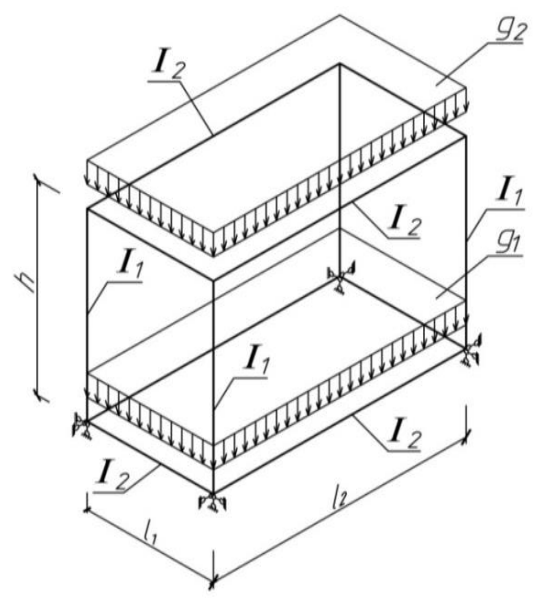

Fig. 1. Design model of the block-module

The wind load on buildings and structures is defined as the sum of the average and pulsation components:

$$
w=w_{m}+w_{p}
$$

Standard value of wind load average component is determined by the formula:

$$
w_{m}=w_{0} \cdot k_{\left(z_{e}\right)} \cdot c
$$

where $w_{0}$ is the standard value of wind load;

$k(z e)$ is a coefficient that takes into account wind load variation height along;

$c$ - aerodynamic coefficient.

Average component of the wind load depends on wind area, terrain and geometry of the building. Therefore, in general, the definition of this component does not any cause difficulties.

Inertial force from the wind load effect on $i$ - form of vibration is determined by:

$$
w_{i}^{p}=m \cdot \xi_{i} \cdot \psi_{i} \cdot y_{i}
$$


where $m$ is the construction mass at $z$ level, referred to surface area to which the wind load is applied;

$\xi i$ is the dynamic coefficient calculated for the period of $i$ - form natural vibrations;

$y i$ is the horizontal construction movement at $z$ level in $i$-form natural vibrations.

$\psi i$ is the coefficient determined by dividing the construction into $r$ sections, within which wind load is assumed to be constant, according to formula

$$
\psi_{i}=\sum_{k=1}^{r} y_{k i} \cdot w_{p k} / \sum_{k=1}^{r} y_{k i}^{2} \cdot M_{k}
$$

where $M_{k}$ is the mass of construction $k$ section;

$y_{k i}$ - horizontal center displacement of $k$ section at $i$ - form vibrations;

$w_{p k}$ - pulsating component resultant of wind load on $k$ section of the construction;

$$
w_{p k}=w_{m} \cdot \zeta \cdot v
$$

To determine the pulsating component of wind load, a technique for the analytical determination of natural vibration frequency was proposed in [10], based on the general dependences [11], according to which the first frequency of a single-storey modular building is equal to:

$$
f=\frac{1}{2 \cdot \pi} \cdot \sqrt{\frac{24 \cdot E \cdot I_{1} \cdot g}{G \cdot h^{3} \cdot\left(1+\frac{I_{1} \cdot l_{2}}{l_{2} \cdot h}\right)}}
$$

where $G$ is the mass collected at one slab point;

$k$ is stiffness coefficient;

$g-$ free fall acceleration.

The equation for determining natural vibration frequency of a multi-storey building has the following form:

$$
f=\frac{1}{2 \cdot \pi} \cdot \sqrt{\frac{24 \cdot E \cdot I_{1} \cdot g}{d \cdot G_{1} \cdot h^{3} \cdot\left(1+\frac{I_{1} \cdot l_{2}}{l_{2} \cdot h}\right)}}
$$

where the coefficient $d$ describes the sum of load ratio:

$$
d=\frac{G_{1}}{G_{1}}+\frac{G_{2}}{G_{1}}+\frac{G_{3}}{G_{1}}+\ldots+\frac{G_{n}}{G_{1}}
$$

here $G_{l}$ is the load on arbitrary cross- beam;

$G_{2}, G_{3}, \ldots G_{n}$ - the load on cross- beams left.

Movements according to $i$ - form oscillations for a single-storey and multi-storey building are determined, respectively

$$
\begin{gathered}
\Delta=\frac{1}{E \cdot I_{1}} \cdot \frac{P \cdot h^{3}}{24}+\frac{1}{E \cdot I_{1}} \cdot \frac{P \cdot l_{2} \cdot h^{2}}{24}=\frac{P \cdot h^{3}}{24 \cdot E \cdot I_{1}} \cdot\left(1+\frac{I_{1} \cdot l_{2}}{I_{2} \cdot h}\right) \\
\Delta=\Delta_{1}+\Delta_{2}+\Delta_{3}=\frac{d \cdot P \cdot h^{3}}{24 \cdot E \cdot I_{1}} \cdot\left(1+\frac{I_{1} \cdot l_{2}}{I_{2} \cdot h}\right)
\end{gathered}
$$

Equations (5) - (10) are obtained on the basis of the following assumptions:

1. All the elements connection of the block (pillars and horizontal frames) with each other are accepted to be stiff.

2. Block coupling with foundations is considered to be hinged-fixed at the angles of the blocks $[12,13]$.

3. Block coupling with each other is assumed to be hinged at the angular points of horizontal frames $[14,15]$.

4. The slab disk is not deformed in its plane. 


\subsection{Forces determination in modular building elements of against wind load}

To determine the wind load forces from the wind impact, it is necessary to consider the direct frame of the block-module, which is acted upon by load uniformly distributed on the pillar (static component) and concentrated in the upper unit (pulsation component) According to the principle of superposition in the frame elements can be defined as the sum of forces from two influences (Figure 2).
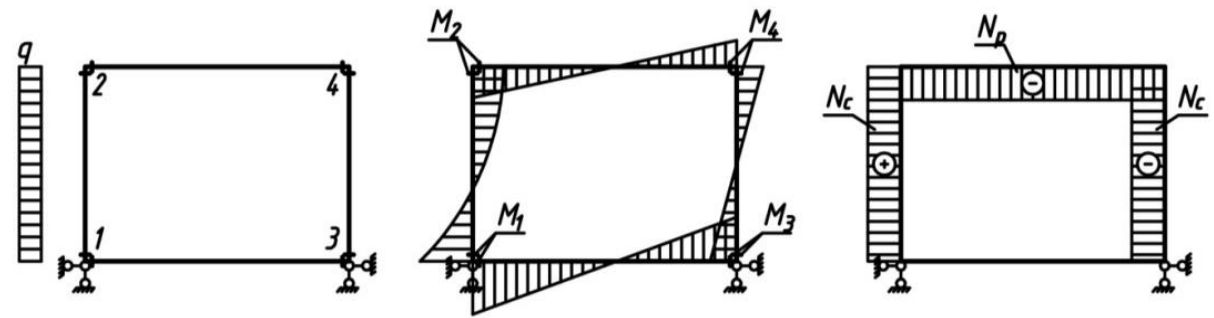

a)
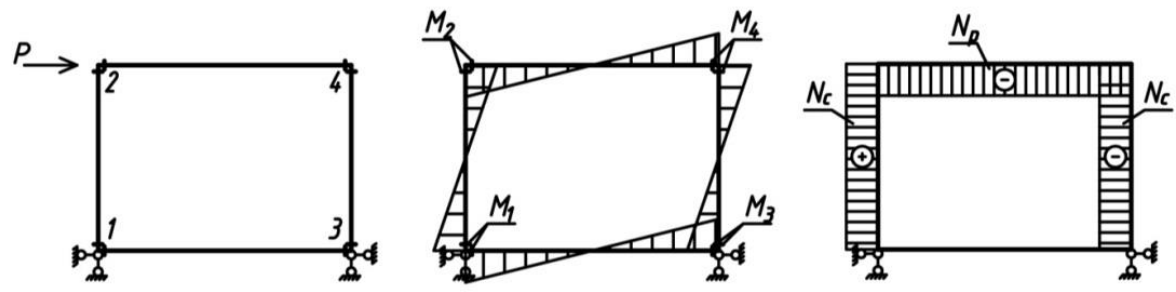

b)

Fig. 2. Calculation scheme and force diagrams in the block-module frame elements: a - from the uniformly distributed load; $\mathrm{b}$ - from the concentrated load

In case of point load acting on the upper unit using structural mechanics methods, it is possible to obtain exact values of bending moments and direct forces in the frame elements. Maximum values of the moments are:

$$
M_{1}=M_{2}=M_{3}=M_{4}=\frac{P \cdot h}{4}
$$

Axial forces in the cross-beam and pillars, respectively:

$$
\begin{gathered}
N_{p}=\frac{P}{2}, \\
N_{c}=\frac{P \cdot h}{4 \cdot l}
\end{gathered}
$$

Under the uniformly distributed wind load on the frame pillar, the exact values of bending moments have an extremely complex shape, since they vary according to a rather complex law and depend on the stiffness ratio of crossbeam and pillar. Such a solution is inconvenient for application in practice; that's why it is necessary to obtain a more convenient expression by approximating the equation of support moment variation of the pillar.

To obtain the approximating function, let us numerically determine bending moments values for frames differing from each other only by pillar stiffness and crossbeam ratios. Frame size is $4 \times 4 \mathrm{~m}$, pillar load is $\mathrm{q}=1 \mathrm{t} / \mathrm{m}$. A graph of bending moment values variation in the lower section of the pillar acted upon by distributed load is given in Figure 3. 


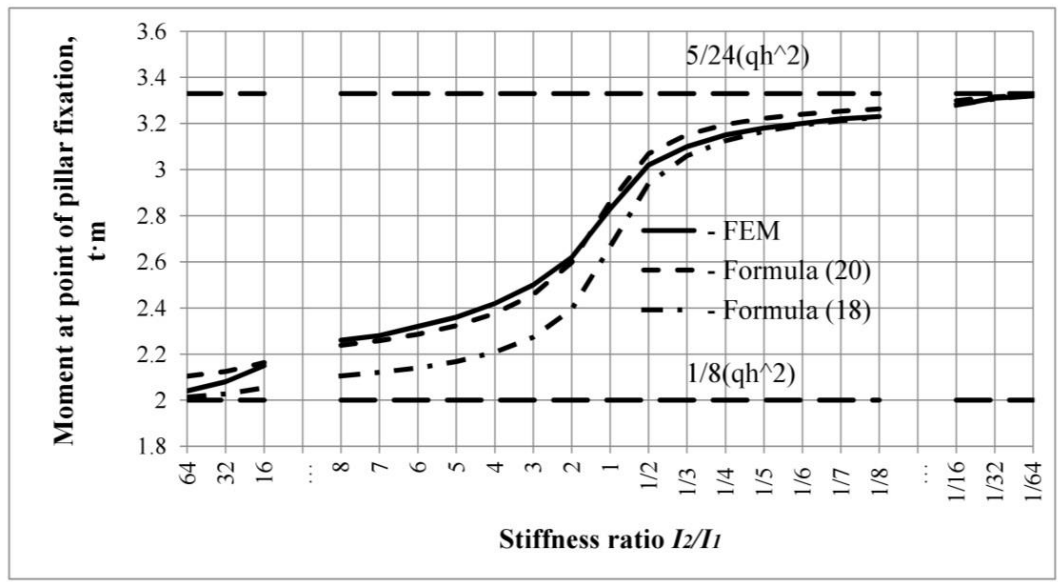

Fig. 3. Graph of moments values variation, depending on the pillar and crossbeam stiffness ratio.

As an approximating function, we take the following:

$$
y=A \cdot \operatorname{arctg}(x)+B
$$

Here, $x=I_{1} \cdot l / I_{2} \cdot h$ - is pillar and crossbeam stiffness ratio;

$A, B$ are coefficients determined by boundary conditions.

To determine the constant coefficients, we should note that with infinite crossbeam stiffness, the value of moment at point of fixation in the lower section of pillar tends to the following value $M_{l}=1 / 8 \cdot\left(q \cdot h^{2}\right)$, at infinite stiffness of $M_{l}=5 / 24 \cdot\left(q \cdot h^{2}\right)$. Bending function point is stiffness ratio $\mathrm{I}_{2} / \mathrm{I}_{1}=1$, which corresponds to the value of moment at point of fixation $M_{1}=4 / 24 \cdot\left(q \cdot h^{2}\right)$.

Given these conditions, equations for determining the $A$ and $B$ coefficients are derived.

At $x=\infty: \operatorname{arctg}(\infty)=\pi / 2 ; y=5 / 3 \cdot\left(q \cdot h^{2} / 8\right)$,

$$
\frac{5}{3} \cdot \frac{q \cdot h^{2}}{8}=A \cdot \frac{\pi}{2}+B
$$

At $x=1: \operatorname{arctg}(1)=\pi / 4 ; y=4 / 3 \cdot\left(q \cdot h^{2} / 8\right)$,

$$
\frac{4}{3} \cdot \frac{q \cdot h^{2}}{8}=A \cdot \frac{\pi}{4}+B
$$

Solving equations (15) and (16), we get

$$
\begin{gathered}
A=\frac{4}{3} \cdot \frac{q \cdot h^{2}}{8 \cdot \pi} \\
B=\frac{q \cdot h^{2}}{8}
\end{gathered}
$$

As a result, moment equation in the lower pillar section will have the following form:

$$
M_{1}=\frac{q \cdot h^{2}}{8}\left(1+\frac{4}{3 \cdot \pi} \cdot \operatorname{arctg}\left(\frac{I_{1} \cdot l}{I_{2} \cdot h}\right)\right)
$$

Expression (19) has rather a simple form and is convenient for engineering applications, while the error in determining the moments values is $10 \%$, that shows a sufficient accuracy of approximation. More accurate approximation can be found adopting form function:

$$
y=A \cdot \operatorname{arctg}(C \cdot x+D)+B
$$

The coefficients A and B are defined above (17) and (18), the coefficients C and D can be calculated by successive selection method. More exact solution has the following form: 


$$
M_{1}=\frac{q \cdot h^{2}}{8}\left(1+\frac{4}{3 \cdot \pi} \cdot \operatorname{arctg}\left(1,5 \cdot \frac{I_{1} \cdot l}{I_{2} \cdot h}+0,1\right)\right)
$$

Absolute values sum of moments in the lower and upper pillar sections are equal, hence it's easy to find the expression for the moment at the pillar top:

$$
M_{2}=\frac{q \cdot h^{2}}{8}\left(1-\frac{4}{3 \cdot \pi} \cdot \operatorname{arctg}\left(1,5 \cdot \frac{I_{1} \cdot l}{I_{2} \cdot h}+0,1\right)\right)
$$

When determining the moments in unloaded pillar, it is acceptable to assume that half of the resultant wind load is transmitted through the upper crossbeam $\mathrm{q} \cdot \mathrm{h} / 2$, then:

$$
M_{3}=M_{4}=\frac{q \cdot h^{2}}{8}
$$

Axial force in pillars is numerically equal to shear force in the crossbeam, consequently:

$$
N_{c}=\frac{q \cdot h^{2}}{8 \cdot l}\left(2-\frac{4}{3 \cdot \pi} \cdot \operatorname{arctg}\left(1,5 \cdot \frac{I_{1} \cdot l}{I_{2} \cdot h}+0,1\right)\right)
$$

Similarly, we obtain the direct force in the crossbeam:

$$
N_{p}=-\frac{q \cdot h}{4}
$$

\section{Calculation results}

To confirm proposed methodology, a modular building consisting of a block of the following dimensions $3 \times 6 \times 4$ (h) $\mathrm{m}$ is considered. Inertia moment of pillars is assumed to be equal to $416,7 \mathrm{~cm}^{4}$ and crossbeam to $236,3 \mathrm{~cm}^{4}$, the vertical load on the floors is accepted to be $p=100 \mathrm{~kg} / \mathrm{m}^{2}$. While calculating, values of maximum bending moment at pillar bearing were determined by proposed method and FEM for standard wind loads corresponding to Russian design standards. Calculation results are shown in Figure 4 and Table 1. Figure 5 and Table 2 show the results of bending moment determining in pillar bearing of the module having the same characteristics for different number of storeys in a building at wind load $w_{0}=38 \mathrm{~kg} / \mathrm{m}^{2}$.

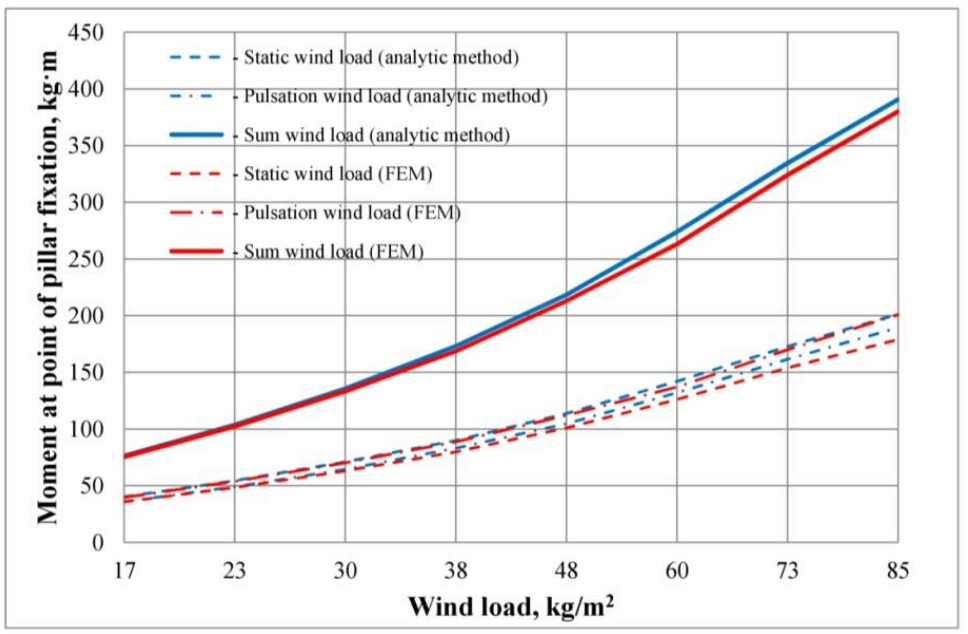

Fig. 4. The results of bending moment determination at pillar bearing using analytical method and FEM for different values of wind loads 
Table 1. The results of bending moment determination at pillar bearing for different values of wind loads

\begin{tabular}{|l|c|c|c|c|c|c|c|c|}
\hline \multicolumn{1}{|c|}{ Wind load, $\mathbf{~ k g ~} / \mathbf{m}^{\mathbf{2}}$} & $\mathbf{1 7}$ & $\mathbf{2 3}$ & $\mathbf{3 0}$ & $\mathbf{3 8}$ & $\mathbf{4 8}$ & $\mathbf{6 0}$ & $\mathbf{7 3}$ & $\mathbf{8 5}$ \\
\hline \multicolumn{8}{|c|}{ Moment values (analytical method) } \\
\hline Static component, $\mathrm{kg} \mathrm{m}$ & 40.3 & 54.5 & 71.0 & 90.0 & 113.6 & 142.1 & 172.8 & 201.2 \\
\hline Pulsation component, kg m & 36.0 & 49.0 & 64.5 & 83.1 & 104.7 & 131.9 & 161.6 & 189.4 \\
\hline Sum value, kg m & 76.3 & 103.5 & 135.5 & 173.1 & 218.3 & 273.9 & 334.4 & 390.6 \\
\hline \multicolumn{8}{|c|}{ Moment values (FEM) } \\
\hline Static component, kg m & 35.8 & 48.4 & 63.1 & 79.9 & 101.0 & 126.0 & 154.0 & 179.0 \\
\hline Pulsation component, kg m & 39.7 & 53.8 & 70.1 & 88.7 & 112.0 & 137.0 & 170.0 & 201.0 \\
\hline Sum value, kg m & 75.5 & 102.2 & 133.2 & 168.6 & 213.0 & 263.0 & 324.0 & 380.0 \\
\hline \multicolumn{8}{|c|}{ Error } \\
\hline Static component, \% & 12.4 & 12.5 & 12.6 & 12.6 & 12.5 & 12.7 & 12.2 & 12.4 \\
\hline Pulsation component, \% & -9.3 & -8.9 & -8.0 & -6.3 & -6.5 & -3.7 & -5.0 & -5.8 \\
\hline Sum value, \% & 1 & 1.2 & 1.7 & 2.7 & 2.5 & 4.2 & 3.2 & 2.8 \\
\hline
\end{tabular}

Analyzing the results, it can be seen that the proposed technique slightly overestimates the bending moments from the static wind load (up to 13\%) and underestimates the pulsation one (up to $10 \%$ ). However, the error in determining sum value, which is taken into account in calculations, does not exceed $5 \%$, and it is considered to be good convergence for engineering calculations. It is also worth noting that when increasing the number of storeys in a building, the error in determining the load is reduced. It happens primarily due to the method of inertial forces determination, which assumes a cantilever design model of the structure, and its accuracy depends on a number of sections which the structure is broken into.

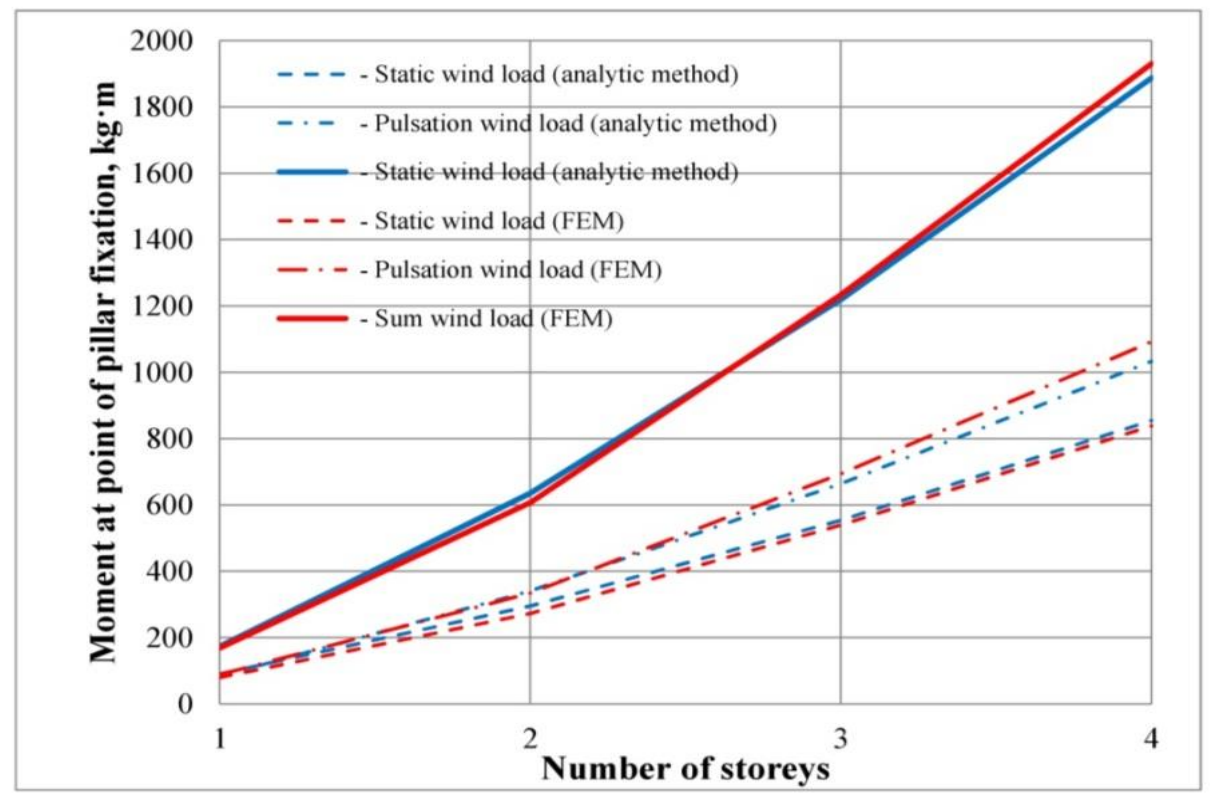

Fig. 5. The results of bending moment determination at pillar bearing using analytical method and FEM for different storeys of the building 
Table 2. The results of bending moment determination at pillar for different number of storeys

\begin{tabular}{|c|c|c|c|c|}
\hline Number of storeys in a building & 1 & 2 & 3 & 4 \\
\hline \multicolumn{5}{|c|}{ Moment values (analytical method) } \\
\hline Static component, kg m & 90.0 & 294.9 & 554.1 & 854.2 \\
\hline Pulsation component, kg m & 83.1 & 340.4 & 664.4 & 1033.2 \\
\hline Sum value, $\mathrm{kg} \mathrm{m}$ & 173.1 & 635.4 & 1218.5 & 1887.3 \\
\hline \multicolumn{5}{|c|}{ Moment values (FEM) } \\
\hline Static component, $\mathrm{kg} \mathrm{m}$ & 79.9 & 273.0 & 539.0 & 838.0 \\
\hline Pulsation component, $\mathrm{kg} \mathrm{m}$ & 88.7 & 334.0 & 694.0 & 1092.0 \\
\hline Sum value, $\mathrm{kg} \mathrm{m}$ & 168.6 & 607.0 & 1233.0 & 1930.0 \\
\hline \multicolumn{5}{|c|}{ Error } \\
\hline Static component, \% & 12.6 & 8.0 & 2.8 & 1.9 \\
\hline Pulsation component, $\%$ & -6.3 & 1.9 & -4.3 & -5.4 \\
\hline Sum value, $\%$ & 2.7 & 4.7 & -1.2 & -2.2 \\
\hline
\end{tabular}

\section{Conclusions}

1. Possibility to use the method at the stage of pre-project decision making, as it does not require compiling a separate calculation scheme in the program complex;

2. The method has a good convergence with the results of numerical force determination by finite element method. The error does not exceed 5\%;

3. The method has a direct connection of building geometry, stiffness characteristics of elements and vibration frequencies;

4. The method allows implementing optimal design of modular buildings structures.

\section{References}

1. R.E. Smith, Prefab architecture: a guide to modular design and construction (Hoboken, New Jersey: John Wiley \& Sons, Inc., 2010)

2. M.F. Musa, M.F. Mohammad et al., Procedia - Soc. and Beh. Sci. 222, 83-92 (2016)

3. L.Y. Dyachenko, O.S. Dyachenko, A.S. Malashenko, Bulletin of PSACEA 2, 69-74 (2016)

4. A.N. Asaul, Yu.N. Kazakov et al., Theory and practice of using prefab buildings in usual conditions and emergency situation in Russia and abroad (St. Petersburg, Gumanistika, 2004)

5. A.N. Mushinskiy, S.S. Zimin, Constr. of unique build. and struct. 4, 182-193 (2015)

6. S.I. Zavrazhnov, D.S. Rachkov et al., Vestnik MGSU 3, 185-190 (2010)

7. A.W. Lacey, W. Chen et al., J. of building eng. 16, 45-56 (2018)

8. I.S. Kholopov, V.S. Shirokov et al., Ind. and civil engin. 6, 15-19 (2015)

9. I.S. Kholopov, V.S. Shirokov, A.V. Solovev, Bull. of LGTU 4, 56-62 (2015)

10. V.S. Shirokov, I.S. Kholopov, A.V. Solovjov, Procedia engin. 153, 655-661 (2016)

11. S.P. Timoshenko Vibration problems in engineering (Moskow, Head redaction of physic-mathematic literature of publishing house "Nauka", 1967)

12. K.-S. Park, J. Moon et al., Engin. structures 110, 244-257 (2016)

13. V.Yu. Alpatov, A.O. Lukin, A.A. Saharov, Ind. and civil engin. 9, 9-14 (2015)

14. Z. Chen, J. Liu, Y. Yu, Engin. structures 147, 625-638 (2017)

15. V.Yu. Alpatov, D.I. Zhuchenko, A.O. Lukin, Vestnik MGSU 2, 142-149 (2017) 